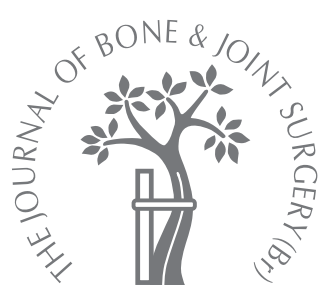

P. P. Kotwal, S. A. Khan

From the All India Institute of Medical Sciences, New Delhi, India
P. P. Kotwal, MBBS, MSOrth, FNA, Professor \& Head - S. A. Khan, MSOrth, DNBOrth, MRCS(Ed), FRCS(Glasgow),

MChOrth(Liverpool) Assistant Professor

Department of Orthopaedics All India Institute of Medica Sciences, Ansari Nagar, New Delhi 110029, India.

Correspondence should be sent to Dr S. A. Khan; e-mail: shahalamkhan@rediffmail.com

(C)2009 British Editorial Society of Bone and Joint Surgery doi:10.1302/0301-620X.91B8. $22074 \$ 2.00$

$J$ Bone Joint Surg [Br] 2009;91-B:1054-7. Received 18 November 2008; Accepted after revision 18 March 2009

\title{
Tuberculosis of the hand
}

\author{
CLINICAL PRESENTATION AND FUNCTIONAL OUTCOME IN 32 \\ PATIENTS
}

A prospective series of 32 cases with tuberculosis of the hand and wrist is presented. The mean age of the patients was 23.9 years ( 3 to 65 ), 12 had bony disease and 20 primarily softtissue involvement. The metacarpal of the little finger was the most commonly involved bone. Pain and swelling were the usual presenting features and discharging sinuses were seen in three cases.

All patients were given anti-tubercular chemotherapy with four drugs. Operative treatment in the form of open or arthroscopic debridement, or incision and drainage of abscesses, was performed in those cases where no response was seen after eight weeks of ATT. Hand function was evaluated by the modified score of Green and O'Brien. The mean was 58.3 (25 to 80 ) before treatment and 90.5 (80 to 95 ) at the end. The mean follow-up was for $\mathbf{2 2 . 4}$ months ( 6 to 43 ). Conservative treatment was successful in 24 patients (75\%). Eight who did not respond to chemotherapy within eight weeks required surgery.

Although tuberculosis of hand has a varied presentation, the majority of lesions respond to conservative treatment.

Although rare, tuberculosis of the hand has a varied clinical presentation, with involvement of bone and soft tissues. ${ }^{1-3}$ There are few reports of its clinical presentation and treatment options and, as increasing numbers of atypical tuberculous presentations are being recognised with the advent of HIV, there is a need for better understanding of these cases. Diagnosis is usually difficult in the early stages, thereby losing time, which can affect the outcome. We present the management of 32 cases of tuberculosis of the hand and wrist.

\section{Materials and Methods}

This was a prospective observational study undertaken between July 2003 and July 2007 in a tertiary referral hospital. Patients attending with suspected tuberculosis of the hand and wrist were included. Those with a past history of operative treatment were excluded. There were 19 males and 13 females with a mean age of 23.9 years (3 to 65). A detailed history and examination were recorded using a special proforma. A full blood count, ESR, and HIV testing by ELISA (enzyme-linked immunosorbent assay) were performed following informed consent. Anteroposterior and lateral radiographs of the hand and wrist were taken in all cases. MRI was undertaken in patients with suspected tubercular tenosynovitis of the flexor/extensor tendons and in those with an undiagnosed soft-tissue swelling in the palm or wrist (Fig. 1). All patients underwent chest radiography to exclude healed or active pulmonary tuberculosis. A core biopsy was done in cases having an obvious bony lesion on a plain radiograph, and soft-tissue lesions underwent fine needle aspiration. A diagnosis of tuberculosis was made on the basis of characteristic histopathology, a positive culture for Mycobacterium tuberculosis and/or a positive smear for acid-fast bacilli. Polymerase chain reaction to detect Mycobacterium tuberculosis was used in equivocal cases. All patients were treated with home-based anti-tubercular chemotherapy (ATT), using four drugs (rifampicin, isoniazid, pyrazinamide and ethambutol) for four months, three drugs (isoniazid, rifampicin and pyrazinamide) for three months and two drugs (isoniazid and rifampicin) for 11 months, according to our standard protocol. Splints or plaster slabs were used for a short time in 17 patients to relieve acute symptoms.

Operative treatment by open or arthroscopic debridement (with or without curettage), or incision and drainage for abscesses was undertaken in those where no response was seen after six to eight weeks of ATT. All patients were followed up regularly. 


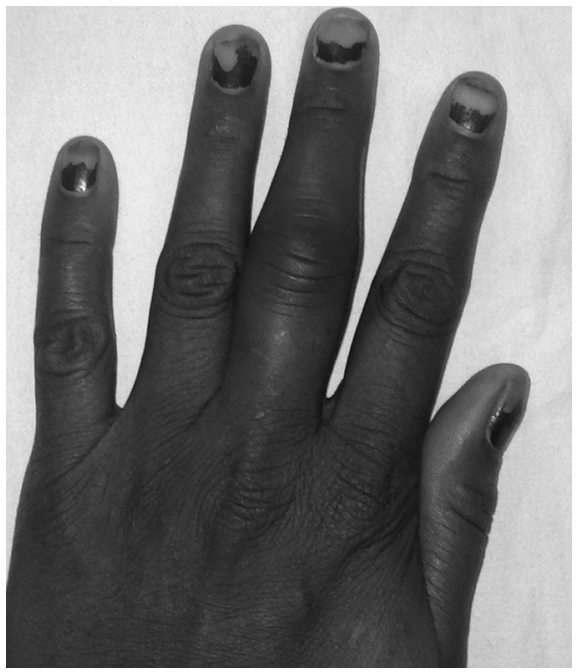

Fig. 1a

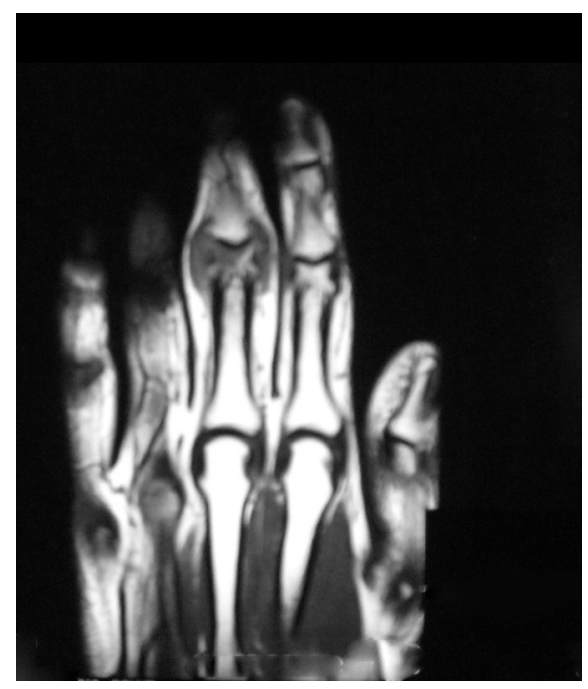

Fig. 1b

a) Clinical photograph showing a patient with tubercular dactylitis of the middle finger. b) MRI of the same patient showing destruction at the proximal interphalangeal joint of the middle finger.

\section{Results}

Based on plain radiographs and MRI, there were 12 patients with bony and 20 with primarily soft-tissue involvement (Table I). The phalanges were primarily involved in four cases, the metacarpals in six and the carpus in two. The fifth metacarpal was the most common metacarpal to be involved (four of the six metacarpal lesions, Figs 2 and 3). The two carpal lesions were in the capitate.

Soft-tissue involvement on MRI was seen as chronic tubercular synovitis of the wrist joint in six patients, flexor tenosynovitis in five, palmar abscess ('compound palmar ganglion') in seven and global involvement of the extensor and flexor tendons in two.
Pain and swelling were the most common presenting features, and discharging sinuses were seen in three cases (Fig. 4). Of the 20 patients with soft-tissue involvement, only three presented with clinical features suggestive of the carpal tunnel syndrome. Of these, two had tubercular synovitis of the wrist and one had flexor tenosynovitis. Nerve conduction studies showed delayed conduction at the carpal tunnel.

Hand function was evaluated using the modified score of Green and O'Brien, ${ }^{4}$ which was recorded in all patients before ATT. The mean score before treatment was 58.3 (25 to 80 ), with patients presenting with a palmar abscess having the lowest scores. No patient had evidence of healed or active pulmonary tuberculosis.

Conservative treatment with ATT was successful in 24 patients $(75 \%)$. The eight who did not respond clinically within three months underwent surgical treatment, three by incision and drainage of a palmar abscess, four by arthroscopic debridement with excision of the diseased synovium of the wrist and one patient with a carpal tunnel syndrome due to flexor tenosynovitis who underwent decompression. All these patients continued their ATT post-operatively. The mean follow-up after completion of ATT was 22.4 months ( 6 to 43 ), no further operations were required and the mean functional score was 90.5 (80 to 95).

\section{Discussion}

Tuberculous tenosynovitis of the hand constitutes less than $1 \%$ of skeletal tuberculosis, ${ }^{5}$ and as in most affections of the hand early diagnosis and treatment are essential for retaining optimal function. The disease is slowly progressive, which causes difficulties in early diagnosis. Late diagnosis leads to poor functional outcome even in the presence of regular ATT. ${ }^{6}$

Pain and swelling are the most common presenting features, followed by discharging sinuses, ${ }^{7}$ as confirmed in our study: discharging sinuses were seen in $9.4 \%$ of our cases. The clinical features depend on the virulence of the organism, the host response and the anatomical area affected. A history of concomitant active pulmonary disease is rarely found with musculoskeletal tuberculosis. Although in a series of 11 patients with tuberculosis of the wrist Benchakroun et $\mathrm{al}^{6}$ described active pulmonary tuberculosis in four cases, there was no case of active or treated pulmonary tuberculosis in our study.

Tuberculosis of the fingers is also known as spina ventosa, but, in order to avoid confusion, it is suggested that all tubercular lesions of the hand should be referred to as 'tuberculosis of the hand'. ${ }^{8}$ Tuberculosis of the fingers is seen typically in the phalanges and interphalangeal joints and is said to be uncommon after five years of age. During childhood these short tubular bones have a lavish blood supply via a nutrient vessel entering the middle of the phalanx in which the first inoculum becomes lodged. ${ }^{7}$ Bony tuberculosis of the phalanges and metacarpals can mimic bone tumours and a histopathological examination is 
Table I. Patient profile, distribution of lesions, treatment and follow-up

\begin{tabular}{|c|c|c|c|c|c|}
\hline $\begin{array}{l}\text { Case } \\
\text { number }\end{array}$ & $\begin{array}{l}\text { Age } \\
\text { (yrs) }\end{array}$ & Gender & Part of hand affected ${ }^{*}$ & Duration of follow-up (mths) & Management \\
\hline 1 & 11 & $M$ & $5^{\text {th }} \mathrm{MC}$ & 19 & Conservative \\
\hline 2 & 13 & $M$ & Palmar abscess & 29 & Conservative \\
\hline 3 & 25 & M & Wrist synovitis & 25 & Conservative \\
\hline 4 & 27 & $\mathrm{~F}$ & Proximal phalanx & 28 & Conservative \\
\hline 5 & 13 & $\mathrm{~F}$ & Wrist synovitis & 32 & Conservative \\
\hline 6 & 17 & M & $4^{\text {th }} \mathrm{MC}$ & 39 & Conservative \\
\hline 7 & 12 & M & Proximal phalanx & 43 & Conservative \\
\hline 8 & 65 & M & Wrist synovitis & 22 & Arthroscopic synovectomy \\
\hline 9 & 19 & $\mathrm{~F}$ & Ext + Flx tenosynovitis & 14 & Conservative \\
\hline 10 & 28 & $\mathrm{~F}$ & Palmar abscess & 15 & Conservative \\
\hline 11 & 32 & $\mathrm{~F}$ & Flx tenosynovitis & 36 & Carpal tunnel decompression \\
\hline 12 & 26 & M & Wrist synovitis & 24 & Conservative \\
\hline 13 & 15 & $\mathrm{~F}$ & Proximal phalanx & 22 & Conservative \\
\hline 14 & 17 & $\mathrm{~F}$ & Palmar abscess & 19 & Incision and drainage \\
\hline 15 & 19 & M & Capitate & 21 & Conservative \\
\hline 16 & 13 & $\mathrm{~F}$ & Palmar abscess & 36 & Conservative \\
\hline 17 & 47 & $\mathrm{~F}$ & Palmar abscess & 29 & Incision and drainage \\
\hline 18 & 10 & M & $5^{\text {th }} \mathrm{MC}$ & 17 & Conservative \\
\hline 19 & 3 & $\mathrm{~F}$ & $5^{\text {th }} \mathrm{MC}$ & 19 & Conservative \\
\hline 20 & 26 & $\mathrm{~F}$ & Ext + Flx tenosynovitis & 9 & Conservative \\
\hline 21 & 55 & M & Wrist synovitis & 14 & Arthroscopic synovectomy \\
\hline 22 & 28 & M & Flx tenosynovitis & 18 & Conservative \\
\hline 23 & 32 & M & Wrist synovitis & 16 & Arthroscopic synovectomy \\
\hline 24 & 9 & M & Capitate & 31 & Conservative \\
\hline 25 & 20 & M & $5^{\text {th }} \mathrm{MC}$ & 32 & Conservative \\
\hline 26 & 26 & M & Palmar abscess & 7 & Conservative \\
\hline 27 & 39 & $\mathrm{~F}$ & Flx tenosynovitis & 27 & Arthroscopic synovectomy \\
\hline 28 & 23 & M & Palmar abscess & 14 & Incision and drainage \\
\hline 29 & 16 & M & Flx tenosynovitis & 19 & Conservative \\
\hline 30 & 19 & M & $1^{\text {st }} \mathrm{MC}$ & 24 & Conservative \\
\hline 31 & 47 & M & Flx tenosynovitis & 6 & Conservative \\
\hline 32 & 12 & $\mathrm{~F}$ & Proximal phalanx & 10 & Conservative \\
\hline
\end{tabular}

* MC, metacarpal; Ext, extensors; Flx, flexors

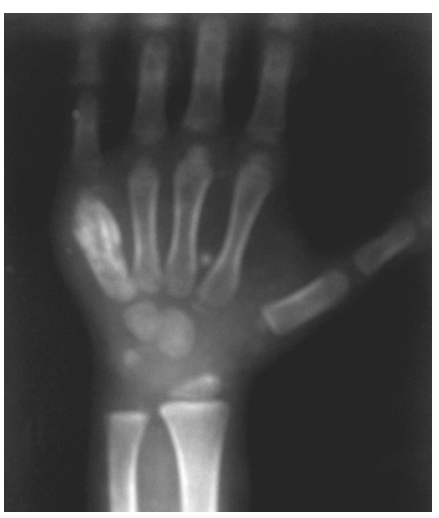

Fig. 2a

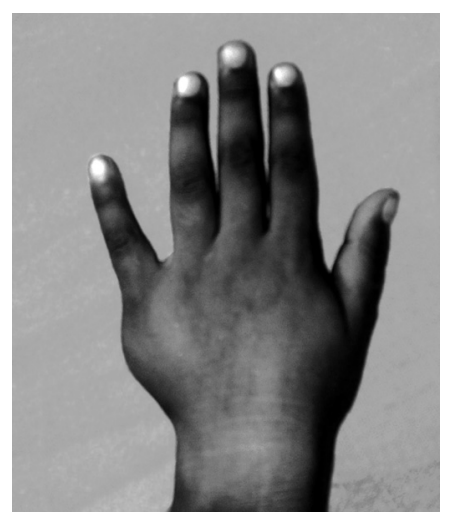

Fig. $2 b$

a) Radiograph of the hand showing sclerosis and new bone formation of the shaft of the fifth metacarpal due to tuberculosis. b) Clinical photograph of the same patient showing the prominent swelling on the ulnar aspect of the hand.

essential for diagnosis. ${ }^{9}$ The functional outcome is better following involvement of thefingers than of the wrist. ${ }^{10,11}$

The treatment of tuberculosis of the hand is generally non-operative. With our wide experience of tubercular osteomyelitis, we feel that surgery should be reserved for specific indications, namely a biopsy, debridement, fusion of a disorganised joint or carpal tunnel decompression. Surgery may also be indicated in a patient who does not 


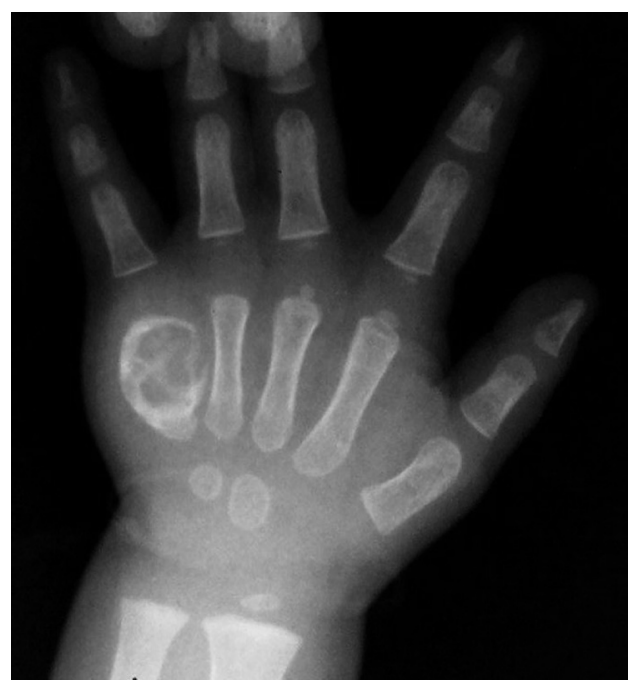

Fig. 3

Radiograph showing a cystic tubercular lesion of the fifth metacarpal in a three-year-old patient (case 19).

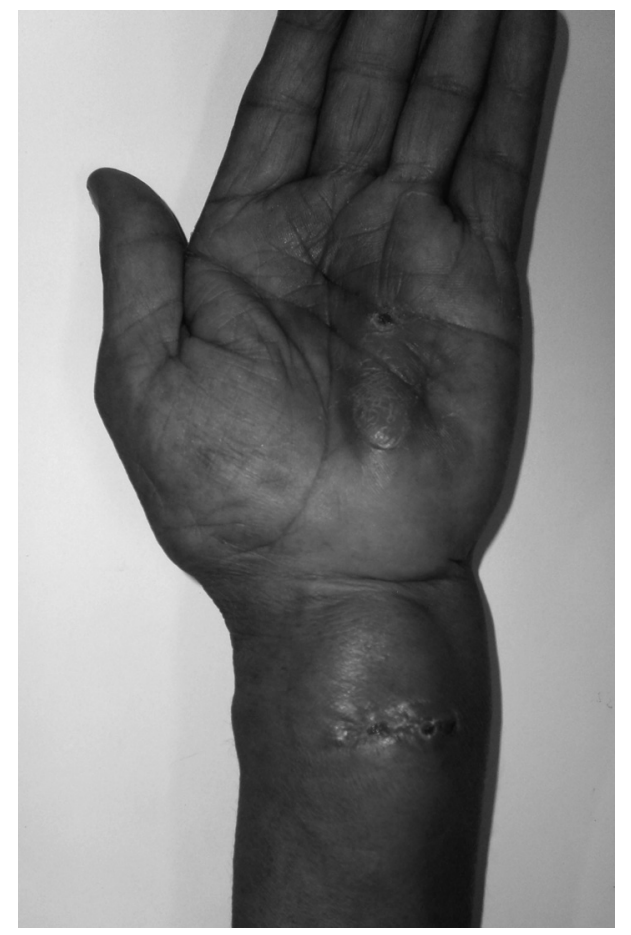

Fig. 4

Clinical picture of a 47-year-old woman with a palmar abscess that extends into the wrist (case 17). initially show a good response to ATT. The local blood flow is increased following surgery, resulting in early healing in musculoskeletal tuberculosis. ${ }^{7}$ Our study shows a good functional outcome in $75 \%$ of cases with conservative home-based management, and similar observations have been made by others. ${ }^{9-11}$

With the worldwide resurgence of musculoskeletal tuberculosis there is an increase in cases affecting atypical sites, including the hand. Further studies with longer follow-up are required to develop early diagnostic tools and treatment protocols to maximise hand function.

No benefits in any form have been received or will be received from a commercial party related directly or indirectly to the subject of this article.

\section{References}

1. Klofkorn RW, Steigerwald JC. Carpal tunnel syndrome as the initial manifestation of tuberculosis. Am J Med 1976;60:583-6.
2. Suso S, Peidro L, Ramon R. Tuberculosis synovitis with "rice bodies" presenting as carpal tunnel syndrome. J Hand Surg [Am] 1988;13:574-6.

3. Lee KE. Tuberculosis presenting as carpal tunnel syndrome. J Hand Surg [Am] 1985;10:242-5.

4. Green DP, O'Brien ET. Open reduction of carpal dislocations: indications and operative techniques. J Hand Surg [Am] 1978;3:250-65.

5. Abdelwahab IF, Bianchi S, Martinoli C, Klein M, Hermann G. Atypical extraspinal musculoskeletal tuberculosis in immunocompetent patients. part II: tuberculosis myositis, tuberculous bursitis, and tuberculous tenosynovites. Can Assoc Radiol J 2006;57:278-86.

6. Benchakroun M, El Bardouni A, Zaddoug 0, et al. Tuberculosis of the wrist: symptoms and outcome in eleven cases. Rev Chir Orthop Reparatrice Appar Mot 2004;90:337-45 (in French).

7. Tuli SM. Tuberculosis of the skeletal system: bone, joints, spine, and bursal sheaths. First ed. New Delhi: Jaypee Brothers Medical Publishers, 1993:116-17.

8. Leung PC. Tuberculosis of the hand. Hand 1978;10:285-91.

9. Subasi M, Bukte Y, Kapukaya A, Gurkan F. Tuberculosis of the metacarpals and phalanges of the hand. Ann Plast Surg 2004;53:469-72.

10. Martini M, Benkeddache Y, Medjani Y, Gottesman H. Tuberculosis of the upper limb joints. Int Orthop 1986;10:17-23.

11. Benkeddache Y, Gottesman H. Skeletal tuberculosis of the wrist and hand: a study of 27 cases. J Hand Surg [Am] 1982;7:593-600. 